International Journal of Engineering \& Technology, $7(2.8)(2018)$ 130-133
International Journal of Engineering \& Technology
SPC
Website: www.sciencepubco.com/index.php/IJET
Research Paper

\title{
A novel approach to improve the efficiency of national rural employment scheme employees using WBASN
}

\author{
P. Rajasekar ${ }^{1} *$, M. Pushpalatha ${ }^{2}$ \\ ${ }^{1}$ Department of Information Technology, SRM University, Kancheepuram, India \\ ${ }^{2}$ Department of Computer Science and Engineering, SRM University, Kancheepuram, India \\ *Corresponding author E-mail: rajasekar.p@ktr.srmuniv.ac.in
}

\begin{abstract}
Health monitoring of human being can be done using implant or wearable sensors. Making use of these sensors to form a network are aptly called as Wireless Body Area Sensor Networks (WBASN). Better performance and energy saving can be accomplished in a challenging way in this type of network. National Rural Employment Guarantee Act in India provides employment for people in rural areas. This involves more intense physical work in the open environment. People involved in this work tend to feel fatigue if they work for prolonged duration. We have conducted experiments in several scenarios to determine the stamina level of employed people and proposed ways to provide how man power can be efficiently improved by giving optimal break time and energy supplement drinks to improve the stamina level. Moreover energy of sensors may be efficiently improved by compressing the data before transmitting them to servers by suitable algorithm as discussed in our paper.
\end{abstract}

Keywords: WBASN; National Employment Guarantee Act; Implant Sensor; Wearable Sensor; Fatigue, Resting Energy Expentidure; Throughput.

\section{Introduction}

WSN attracts many researchers for its various applications and its efficiency. WBASN is a kind of WSN which uses many sensors to measure the human physiological factors like pulse rate, temperature, heartbeat, EEG in real time[1] [12]. In India, our government established National Rural Employment Guarantee Act in 2005. The main purpose of this Act are i) to provide guarantee the right to work ii) to enhance livelihood security in rural areas. iii) to provide 120 days of employment in a financial year. The following tasks are carried out in rural areas i) creating infrastructure of water harvesting ii) drought relief iii) flood control etc., thereby creating rural assets to the nation.

People who are involved in this work are directly exposed to environment and they become weak physically. In this paper, we propose a novel method to determine the energy level of people by WBASN. Decrease in energy level leads to less productivity of work if they are not treated in time by providing rest or supplement drinks to boost their glucose level. In this method we used HEER routing protocol which conserve the energy of nodes in an efficient manner in this operating environment.

The drawback of WBASN are costly sensors, placement of sensors and its stability [2] [3]. Improper placements of nodes leads to low quality data being sent by the sensor. Placement of low powered and tiny sensors is a great concern [4].

\section{Related works}

Network lifetime can be improved by proper data transmission and even distribution as in [5] recommended using Energy balanced rate assignment and routing protocol for BAN. Nature of connection between nodes depends on movements of body. Op- portunistic routing protocol improves the senor life even during body movements as in [6].

Q-LEACH [7] has four quadrant each has a specific number of nodes and they are further divided into sectors. Each sector selects its own $\mathrm{CH}$. This reduces the load on $\mathrm{CH}$.

A. Ahmad et al. proposed Density controlled Divide and Rule scheme [8] for energy efficient routing in WSN. In this method static clustering techniques is used. Routing protocol suggested by N. Javaid. et al [9] for heterogeneous WBASN. In [10] researchers made a detailed survey on various architectures to monitor health status in WBASN.

On comparing the above works, it is found that they are focused on energy efficiency of nodes. In our suggested experiments we propose a method to extend the network life time by introducing a suitable compression method based on experiment results.

\section{Proposed method}

We assume people involved in this work are in groups and each of them are given three different sensors viz., heartbeat sensor, blood glucose level sensor and temperature sensor[19][20]. Heart beat sensor or pulse sensor use non invasive method to take measurements at the skin surface. Optical technique is used to detect blood volume by using photo detector under bed of tissue [13] [14]. It uses an IR to illuminate the finger on one side. Photo detector is placed on other side to measure the small change in variations of transmitted light intensity. Some of the light is absorbed by the blood. Light detector recognizes the transmitted or reflected light to calculate the heart beat. LM35 of Texas Instruments is used to calculate body temperature. Its operating range is between $-55^{\circ} \mathrm{C}$ and $+150^{\circ} \mathrm{C}$. It is suitable for remote applications and it is calibrated directly in Celsius [15] [16]. 
Dexcom G5 sensor is used for real-time continuous glucose monitoring. It monitors the blood glucose level without pricking fingers and approved by Food and Drug Administration(FDA). Data sensed by these sensors are collected by Central Base Unit(CBU) which is placed on the wrist and CBU pushes these information to the server.

Temperature sensor is placed in the armpit to measure the temperature of the person. Blood glucose level sensor is positioned in the finger tip to take blood samples periodically to measure the glucose level in blood. Heartbeat sensor is placed at the finger tip to measure the heartbeat of the person. Alternately they can also be positioned in ear lobe. CBU is placed around wrist of a person as a wearable band [17] [18] [21] [22]. This is used to collect the sensor values continuously which in turn send these information to a remote server as shown in Fig. 1.

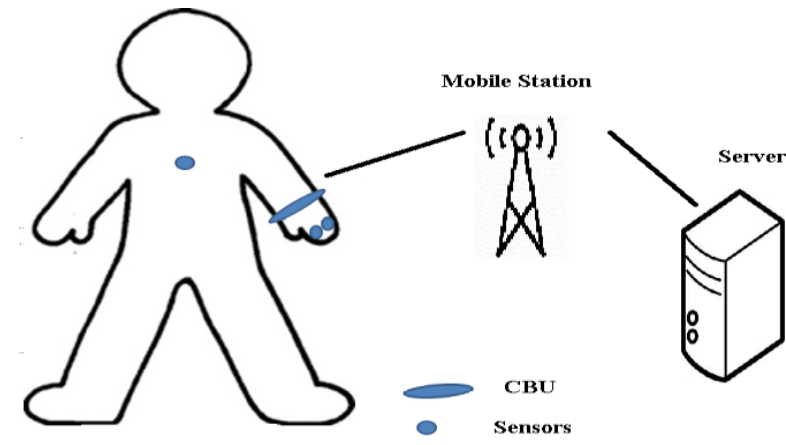

Fig. 1: Sensors on Human Body and Its Communications.

Our experiment is carried out with 40 employees based on sex and age. 20 men and 20 women were involved for this experiment who are classified based on four age groups as follows. People who falls into 20 to 30 years old, 31 to 40 years old, 41 to 50 years old and 51 to 59 years old. Five persons were selected in each age category. All of them are fitted with those three sensors as mentioned above.

Classification followed in our experiment is shown in Fig. 2.

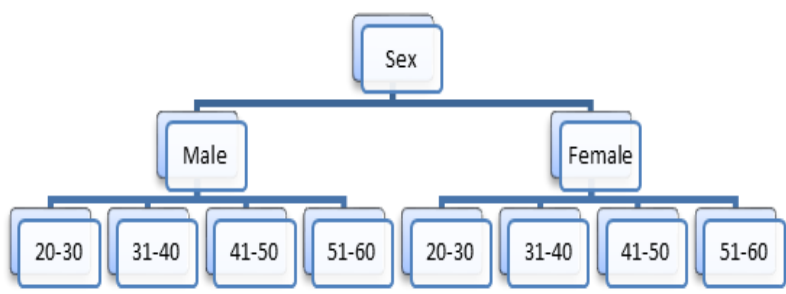

Fig. 2: Classification Based on Age and Sex.

The initial average stamina level of the person is taken as 2500 joules. Stamina level is assumed to be maximum before they start the work. As the time progresses, due to their intense physical work body metabolism changes accordingly. We are concerned in calculating their body temperature, blood glucose level and heart beat with available sensors on body.

We have used Mifflin-St Jeor Formula to calculate the Resting Energy Expenditure (REE).

Mifflin-St Jeor formula for men is given below:

$\mathrm{REE}=10 \mathrm{x}$ weight $(\mathrm{kg})+6.25 \mathrm{x}$ height $(\mathrm{cm})-5 \mathrm{x}$ age $(\mathrm{y})+5$

Mifflin-St Jeor formula for women is given below:

REE $=10 \mathrm{x}$ weight $(\mathrm{kg})+6.25 \mathrm{x}$ height $(\mathrm{cm})-5 \mathrm{x}$ age $(\mathrm{y})-161$

Resting Energy Expenditure (REE) is defined as the amount of energy expended by a person at rest. The initial average stamina level of the person is taken as 2500 joules. This is required for the proper functioning of various vital organs of human body. This minimum energy required for a person is considered as threshold value. If it reaches below 1500 joules, the person is said to have least stamina level.

In our experiment we use energy consumption model in [11] and transmission energy and reception energy is given as below:

Transmission Energy = Energy Dissipated by Transmitter circuitry*b+Transmit Amplifier Energy $* m * b * d m$

Reception Energy $=$ Energy Dissipated by Receiver circuitry $* b$

Where $b$ is the numbers of bits being transmitted. Blood glucose sensor and temperature sensor use 240 bits data while heartbeat sensor use $24 \mathrm{k}$ bits. $\mathrm{m}$ is path loss exponent. For Line of Sight(LOS) communication, its value is 3.3778 and for other non LOS model the value is 5.778 .

\section{Experiment and result}

We used MATLAB[12] to simulate our work. In this model we assumed the initial energy as $0.34 \mathrm{~J}$. Transmitting and receiving energy of transmitter is $16.698 \mathrm{~nJ} / \mathrm{bit}$ and that of receiver is 36 $\mathrm{nJ} / \mathrm{bit}[23][24]$. Amplification energy is taken as $2 \mathrm{~nJ} / \mathrm{bit}^{2} \mathrm{~m}^{2}$. All three sensor readings were taken from $10 \mathrm{am}$ to $6 \mathrm{pm}$ in an interval of one hour and they are tabulated. The number of packets being transmitted is also noted down for each sex. Five people's reading for each age category is taken and they are averaged to reduce error and 95\% confidence level is assumed. Objectives of our experiment are,

- To find who discharges work efficiently over the period of time.

- When to provide break time

- Throughput analysis

- Improving Node lifetime

Fig. 3 shows graph of stamina level of men in various age categories over a time period. It shows that beginning of the day all are in full stamina level. As the time progresses the energy level of the employees goes down. But after the meal break at $1 \mathrm{pm}$ improves their stamina a bit and gradually there after decreases at the end of their days work

\section{Stamina Level - Men}

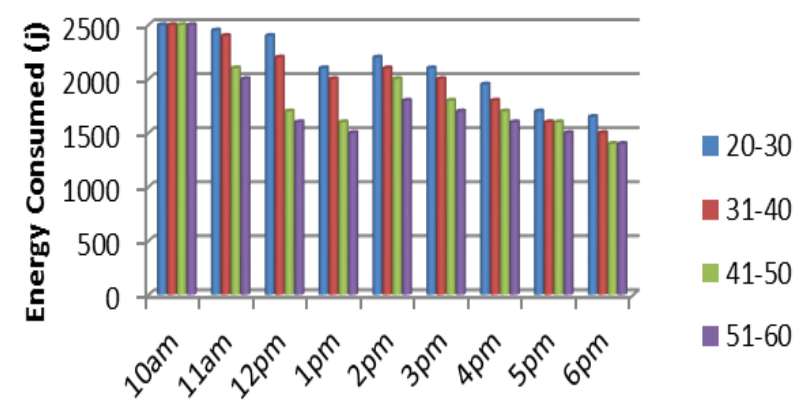

Time Interval

Fig. 3: Stamina Level of Men over A Period.

Fig. 4 shows graph of stamina level of women in various age categories over a time period. It shows that beginning of the day all are in full stamina level. As the time progresses the energy level of the employees goes down. But after the meal break at $1 \mathrm{pm}$ improves their stamina a bit and gradually there after decreases at the end of their days work. Comparing with figure 3 we infer that women tend to reach fatigue so earlier. Moreover contribution by 51-60 age category is less as they reach fatigue soon compared to other categories. It is also noted that as the temperature and heartbeat increases energy level of villagers is drops to threshold. As the glucose level decreases the energy level decreases. They are revived by optimal break time and provided with supplement energy drinks like coconut water, buttermilk etc., for the needy to 
improve their productivity. Unnecessary break time may be eliminated if they found to me fit by analyzing continuously by our method.

\section{Stamina Level - Women}

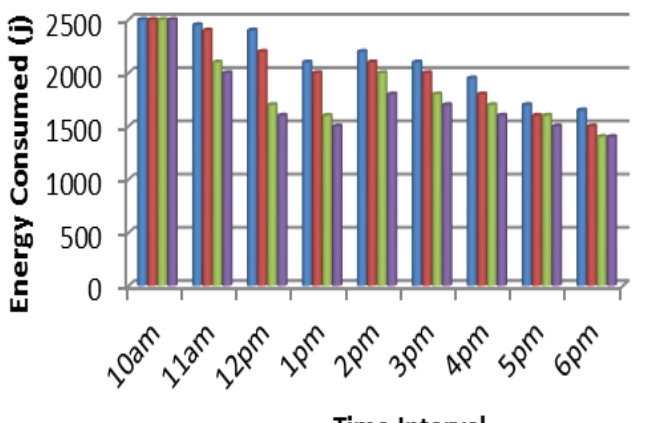

- 20-30

\section{Time Interval}

Fig. 4: Stamina Level of Women over A Period.

Fig. 5. shows the realtion between packets sent over a period of time for men workers. Age group 20-30 very rarely get tired and transmission of data from sensors are less frequent. Hence very few number of packets are sent. But as the age group increases we found there are large number of packets being transmitted to CBU. Sending more packets results in more energy spending by the motes. Network lifetime is more for 20-30 age group and it is less for the other age groups as noticed from this graph.

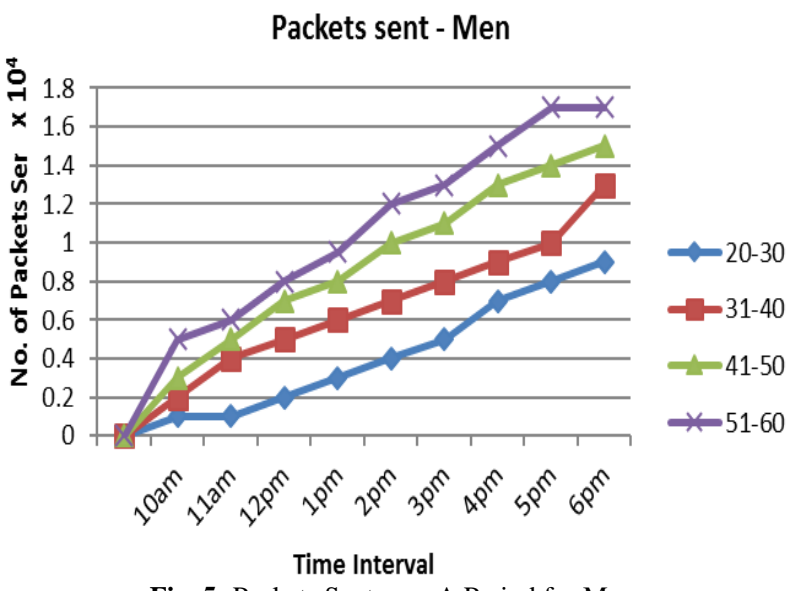

Fig. 5: Packets Sent over A Period for Men.

Fig. 6. shows the realtion between packets sent over a period of time for women workers. Age group 20-30 very rarely get tired and transmission of data from sensors are less frequent. But somehow more packets are sent when compared to men of the same age group.

As the age group increases we found there are large number of packets being transmitted to CBU. Sending more packets results in more energy spending by the motes. Network lifetime is more for 20-30 age group and it is less for the other age groups as noticed from this graph. Energy can be saved when transmitting data for other age group by using appropriate compression algorithm[24] [25] like Huffman encoding, Delta Run length encoding etc.

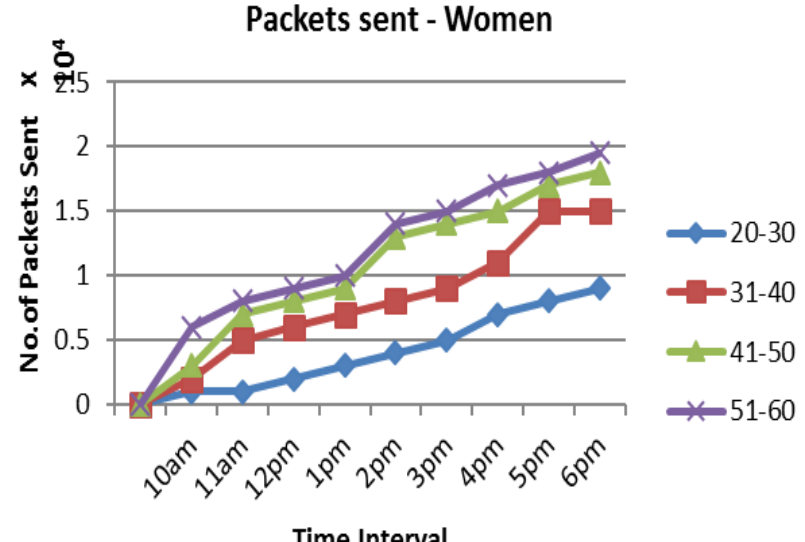

Fig. 6: Packets Sent over A Period For Women.

\section{Conclusion and future work}

Better performance and energy saving can be accomplished in a challenging way in WBASN type of network. Our experimental results show that young people work for long duration with less energy spent. When comparing men with women of same age category, men expected to give more productivity than women. This experiment provides us to determine the optimal break time to be given for various categories of people and identify when to compress the data. Hence man power clustering can be done in an optimal manner. As older people [51-60 age category] gets tired very soon, more packets were transmitted requiring more bandwidth. So proper compression method can be implemented to improve the lifetime of the sensors. However compression algorithm is not needed for low age category [20-30] because of less bandwidth consumption. Incorporating compression for the latter scenario may decrease the lifetime of the sensors due to its complexity at hardware level.

\section{References}

[1] J. M. Maloney and Jr. J. T. Santini, "Implantable microchips for controlled drug delivery," the 26th International Conference of the IEEE Engineering in Medicine and Biology Society, Page(s):2668 2669, September 2004

[2] B.J. Nelson, I.K Kaliakatsos, J. J. Abbott. "Microrobots for minimally invasive medicine" Annu Rev Biomed Eng. 15; 12:55-85, Aug. 2010. https://doi.org/10.1146/annurev-bioeng-010510-103409.

[3] M. R. Yuce, H. C. Keong, and M. Chae, "Wideband communication for implantable and wearable systems," IEEE Transactions on Microwave Theory and Techniques, vol. 57, Part 2, pp. 2597-2604, October 2009. https://doi.org/10.1109/TMTT.2009.2029958.

[4] M. R. Yuce and J. Khan, Wireless Body Area Networks: Technology, Implementation and Applications, Pan Stanford Publishing, ISBN 978-981-431-6712, 2011.

[5] N. Ababneh, N. Timmons, J. Morrison, and D. Tracey, "Energybalanced rate assignment and routing protocol for body area networks", in Ad- vanced Information Networking and Applications Workshops (WAINA), 2012 26th International Conference on. IEEE, 2012, pp. 466471.

[6] Maskooki, C. B. Soh, E. Gunawan, and K. S. Low, "Opportunistic routing for body area network", in Consumer Communications and Networking Conference (CCNC), 2011 IEEE. IEEE, 2011, pp. 237 241.

[7] Manzoor, N. Javaid, O. Rehman, M. Akbar, Q. Nadeem, A. Iqbal, M. Ishfaq, "Q-LEACH: A New Routing Protocol for WSNs", International Workshop on Body Area Sensor Networks (BASNet2013) in conjunction with 4th International Conference on Ambient Systems, Networks and Technologies (ANT 2013), 2013, Halifax, Nova Scotia, Canada, Procedia Computer Science, Volume 19, 2013, Pages 926-931 https://doi.org/10.1016/j.procs.2013.06.127.

[8] Ahmad, K. Latif, N. Javaid, Z. A. Khan and U. Qasim, "Density Controlled Divide-And-Rule Scheme For Energy Efficient Routing In Wireless Sensor Networks", 26th IEEE Canadian Conference on Electrical and Computer Engineering (CCECE2013), Regina, Saskatchewan, Canada, 2013. 
[9] N. Javaid, Z. Abbas, M. S. Farid, Z. A. Khan and N. Alrajeh, "MATTEMPT: A New Energy-Efficient Routing Protocol for Wireless Body Area Sensor Networks", The 4th International Conference on Ambient Systems, Networks and Technologies (ANT 2013), 2013, Halifax, Nova Scotia, Canada, Procedia Computer Science, Volume 19, 2013. https://doi.org/10.1016/j.procs.2013.06.033.

[10] N. A. Khan, N. Javaid, Z. A. Khan, M. Jaffar, U. Rafiq, A. Bibi, "Ubiquitous Healthcare in Wireless Body Area Networks", 2012 IEEE 11th International Conference on Trust, Security and Privacy in Computing and Communications (TrustCom), Liverpool, UK, pp. 1960-1967, 2012.

[11] Bayilmis and M. Younis, "Energy-aware gateway selection for increasing the lifetime of wireless body area sensor networks", Journal of medical systems, vol. 36, no. 3, pp. 15931601, 2012.

[12] MATLAB 7.4.0(R2007a) www.mathworks.com

[13] M. R. Yuce, "Implementation of Wireless Body Area Networks for Healthcare Systems," Sensors \& Actuators: A. Physical, vol. 162, pp. 116-129, July 2010. https://doi.org/10.1016/j.sna.2010.06.004.

[14] M. R. Yuce and J. Khan, Wireless Body Area Networks: Technology, Implementation and Applications, Pan Stanford Publishing, ISBN 978-981-431-6712, 2011.

[15] Y.Gao, et al., "Low-power ultra-wideband wireless telemetry transceiver for medical sensor applications," IEEE Transactions on Biomedical Engineering, vol.58, pp.768-772, March 2011. https://doi.org/10.1109/TBME.2010.2097262.

[16] Ho Chee Keong and M. R. Yuce, "UWB-WBAN sensor node design," the international Conference of the IEEE Engineering in Medicine and Biology Society, pp. 2176 - 2179 Aug. 30 2011-Sept. 32011 .

[17] K. Sonoda, et al., "Wearable photoplethysmographic sensor system with PSoC microcontroller," Fifth International Conf. on Emerging Trends in Engin. and Technology (ICETET), pp. 61-65, 2012. https://doi.org/10.1109/ICETET.2012.19.

[18] Z. Y. Wang, V. Leonov, P. Fiorini, and C. V. Hoof, "Realization of a wearable miniaturized generator for human body applications," Sensors and actuators: A, vol. 156, pp. 95-102, 2009. https://doi.org/10.1016/j.sna.2009.02.028.

[19] R. M. Shubair and H. Elayan, "In vivo wireless body communications: State-of-the-art and future directions," in Antennas \& Propagation Conference (LAPC), 2015 Loughborough. IEEE, 2015, pp. $1-5$.

[20] D. Patron, K. Gedin, T. Kurzweg, A. Fontecchio, G. Dion, and K. R. Dandekar, "A wearable rfid sensor and effects of human body proxim-ity," in 2014 IEEE Benjamin Franklin Symposium on Microwave and Antenna Sub-systems for Radar, Telecommunications, and Biomedical

[21] H. Fotouhi, A. Causevic, M. Vahabi, and M. Bjrkman, "Interoperability in heterogeneous low-power wireless networks for health monitoring systems," in 2016 IEEE International Conference on Communications Workshops (ICC), May 2016, pp. 393-398. https://doi.org/10.1109/ICCW.2016.7503819.

[22] M.Berger. (2013) "Nanotechnology for implantable sensors", nanowerk.com.

[23] M. Pacelli, G. Loriga, N. Taccini, and R. Paradiso, "Sensing fabrics for monitoring physiological and biomechanical variables: E-textile solutions," in Proceedings of the 3rd IEEE-EMBS, 2006, pp. 1-4.

[24] C. Pang et al., "Recent advances in flexible sensors for wearable and implantable devices," J. Appl. Polym. Sci., vol. 130, pp. 14291441, Nov. 2013. https://doi.org/10.1002/app.39461.

[25] B. Ying, Y. Liu, H. Yang, and H. Wang, "Evaluation of tunable data compression in energy-aware wireless sensor networks," Sensors, vol. 10, no. 4, pp. 3195-217, 2010. https://doi.org/10.3390/s100403195.

[26] Y. Liang, "Efficient temporal compression in wireless sensor networks," in IEEE the 36th Conference on Local Computer Networks (LCN), Bonn, Germany, 2011, pp.466-474. https://doi.org/10.1109/LCN.2011.6115508.

[27] S.V.Manikanthan and V.Rama“Optimal Performance of Key Predistribution Protocol In Wireless Sensor Networks" International Innovative Research Journal of Engineering and Technology, ISSN NO: 2456-1983, Vol-2, Issue -Special-March 2017.

[28] T. Padmapriya and V. Saminadan, "Inter-cell Load Balancing Technique for Multi- class Traffic in MIMO - LTE - A Networks", International Conference on Advanced Computer Science and Information Technology, Singapore, vol.3, no.8, July 2015.

[29] Meka Bharadwaj, Hari Kishore "Enhanced Launch-Off-Capture Testing Using BIST Designs" Journal of Engineering and Applied Sciences, ISSN No: 1816-949X, Vol No.12, Issue No.3, page: 636643, April 2017. 